\title{
GENERALIZED KELLER-SEGEL MODELS OF CHEMOTAXIS. ANALOGY WITH NONLINEAR MEAN FIELD FOKKER-PLANCK EQUATIONS
}

\author{
PIERRE-HENRI CHAVANIS $\ddagger$ \\ Laboratoire de Physique Théorique, Université Paul Sabatier, 118 route de Narbonne \\ 31062 Toulouse, France \\ ${ }^{\ddagger}$-mail: chavanis@irsamc.ups-tlse.fr
}

\begin{abstract}
We consider a generalized class of Keller-Segel models describing the chemotaxis of biological populations (bacteria, amoebae, endothelial cells, social insects,...). We show the analogy with nonlinear mean field Fokker-Planck equations and generalized thermodynamics. As an illustration, we introduce a new model of chemotaxis incorporating both effects of anomalous diffusion and exclusion principle (volume filling). We also discuss the analogy between biological populations described by the Keller-Segel model and self-gravitating Brownian particles described by the Smoluchowski-Poisson system.
\end{abstract}

\section{Introduction}

The name chemotaxis refers to the motion of organisms induced by chemical signals [1]. In some cases, the biological organisms (bacteria, amoebae, endothelial cells, ants...) secrete a substance (pheromone, smell, food, ...) that has an attractive effect on the organisms themselves. Therefore, in addition to their diffusive motion, they move preferentially along the gradient of concentration of the chemical they secrete (chemotactic flux). When attraction prevails over diffusion, the chemotaxis can trigger a self-accelerating process until a point at which aggregation takes place. This is the case for the slime mold Dictyostelium discoideum and for the bacteria Escherichia coli. This is referred to as chemotactic collapse. A model of slime mold aggregation has been introduced by Patlak [2] and Keller \& Segel [3] in the form of two coupled differential equations. The first equation is a drift-diffusion equation describing the evolution of the concentration of bacteria and the second equation is a diffusion equation with terms of source and degradation describing the evolution of the concentration of the chemical. In the simplest model, the diffusion coefficient $D$ and the mobility $\chi$ of the bacteria are con- 
stant. This forms the standard Keller-Segel model. However, the original Keller-Segel model allows these coefficients to depend on the concentration of the bacteria and of the chemical. If we assume that they only depend on the concentration of the bacteria, the general Keller-Segel model becomes similar to a nonlinear mean field Fokker-Planck equation. Nonlinear FokkerPlanck (NFP) equations have been introduced in a very different context, in relation with a notion of generalized thermodynamics [4]. As far as we know, the connection between the general Keller-Segel model and nonlinear mean field Fokker-Planck equations has been first mentioned in Chavanis [5] and developed in subsequent papers (see [6] and references therein). This analogy makes possible to interpret results obtained in chemotaxis in terms of a generalized thermodynamics. At the same time, chemotaxis becomes an example of great physical importance for which a notion of (effective) generalized thermodynamics is justified.

The standard Keller-Segel (KS) model has been extensively studied in the mathematical literature (see the review by Horstmann [7]). It was found early that, above a critical mass, the distribution of bacteria becomes unstable and collapses. This chemotactic collapse leads ultimately to the formation of Dirac peaks [8-20]. Recently, it was shown by Chavanis, Rosier \& Sire [21] that, when the equation for the evolution of the concentration is approximated by a Poisson equation [10,14,17], the standard Keller-Segel (KS) model is isomorphic to the Smoluchowski-Poisson (SP) system describing self-gravitating Brownian particles. The chemotactic collapse of biological populations above a critical mass is equivalent to the gravitational collapse of self-gravitating Brownian particles below a critical temperature [22]. Assuming that the evolution is spherically symmetric, Chavanis \& Sire [21-28] were able to describe all the phases of the collapse (pre-collapse and post-collapse) analytically in $d$ dimensions, including the critical dimension $d=2$.

Recently, some authors have considered generalizations of the standard Keller-Segel (KS) model. Two main classes of generalized Keller-Segel (GKS) models of chemotaxis have been introduced:

(i) Models with filling factor: Hillen \& Painter [29,30] considered a model with a normal diffusion and a density-dependent mobility $\chi(\rho)=$ $\chi\left(1-\rho / \sigma_{0}\right)$ vanishing above a maximum density $\sigma_{0}$. The same model was introduced independently by Chavanis [5,31] in relation with an "exclusion principle" connected to the Fermi-Dirac entropy in physical space. In these models, the density of bacteria remains always bounded by the maximum density: $\rho(\mathbf{r}, t) \leq \sigma_{0}$. This takes into account finite size effects 
and filling factors. Indeed, since the cells have a finite size, they cannot be compressed indefinitely. In this generalized Keller-Segel model, chemotactic collapse leads ultimately to the formation of a smooth aggregate instead of a Dirac peak in the standard Keller-Segel model. This regularized model prevents finite time-blow up and the formation of (unphysical) singularities like infinite density profiles and Dirac peaks. Therefore, the Dirac peaks (singularities) are replaced by smooth density profiles (aggregates).

(ii) Models with anomalous diffusion: Chavanis \& Sire [32] studied a model with a constant mobility and a power law diffusion coefficient $D(\rho)=D \rho^{\gamma-1}$ (with $\gamma=1+1 / n$ ). This lead to a process of anomalous diffusion connected to the Tsallis entropy [33]. For $0<n<n_{3}=d /(d-2)$, the system reaches a self-confined distribution similar to a stable polytrope (e.g. a classical white dwarf star) in astrophysics. For $n>n_{3}$, the system undergoes chemotactic collapse above a critical mass (the classical chemotactic collapse related to the standard Keller-Segel model is recovered for $n \rightarrow+\infty$ ) [32]. In the pre-collapse regime, the evolution is self-similar and leads to a finite time singularity. A Dirac peak is formed in the post-collapse regime. For $n=n_{3}$, the dynamics is peculiar and involves a critical mass similar to the Chandrasekhar limiting mass of relativistic white dwarf stars in astrophysics [34]. The case of negative index $n<0$ is treated in [35] with particular emphasis on the index $n=-1$ leading to logotropes.

In the present paper, we discuss a larger class of generalized Keller-Segel models and interprete these equations in relation with nonlinear mean field Fokker-Planck equations and generalized thermodynamics. For illustration, we present for the first time a model incorpoating both a filling factor and some effects of anomalous diffusion.

\section{The generalized Keller-Segel model}

\subsection{The dynamical equations}

The general Keller-Segel model [3] describing the chemotaxis of bacterial populations consists in two coupled differential equations

$$
\begin{gathered}
\frac{\partial \rho}{\partial t}=\nabla \cdot\left(D_{2} \nabla \rho\right)-\nabla \cdot\left(D_{1} \nabla c\right), \\
\epsilon \frac{\partial c}{\partial t}=-k(c) c+f(c) \rho+D_{c} \Delta c,
\end{gathered}
$$

that govern the evolution of the density of bacteria $\rho(\mathbf{r}, t)$ and the evolution of the secreted chemical $c(\mathbf{r}, t)$. The bacteria diffuse with a diffusion 
coefficient $D_{2}$ and they also move in a direction of a positive gradient of the chemical (chemotactic drift). The coefficient $D_{1}$ is a measure of the strength of the influence of the chemical gradient on the flow of bacteria. On the other hand, the chemical is produced by the bacteria with a rate $f(c)$ and is degraded with a rate $k(c)$. It also diffuses with a diffusion coefficient $D_{c}$. In the general Keller-Segel model, $D_{1}=D_{1}(\rho, c)$ and $D_{2}=D_{2}(\rho, c)$ can both depend on the concentration of the bacteria and of the chemical. This takes into account microscopic constraints, like close-packing effects, that can hinder the movement of bacteria. If we assume that the quantities only depend on the concentration of bacteria and write $D_{2}=D h(\rho)$, $D_{1}=\chi g(\rho), k(c)=k^{2}, f(c)=\lambda$ and $D_{c}=1$, we obtain

$$
\begin{gathered}
\frac{\partial \rho}{\partial t}=\nabla \cdot(D h(\rho) \nabla \rho-\chi g(\rho) \nabla c), \\
\epsilon \frac{\partial c}{\partial t}=\Delta c-k^{2} c+\lambda \rho .
\end{gathered}
$$

For $\epsilon=0$, Eq. (4) becomes the screened Poisson equation

$$
\Delta c-k^{2} c=-\lambda \rho .
$$

Therefore, we can identify $k^{-1}$ as the screening length. If we assume furthermore that $k=0$, we get the Poisson equation

$$
\Delta c=-\lambda \rho .
$$

The generalized Keller-Segel (GKS) model (3) can be viewed as a nonlinear mean-field Fokker-Planck (NFP) equation [5]. Written in the form $\partial_{t} \rho=\nabla \cdot(\nabla(D(\rho) \rho)-\chi(\rho) \rho \nabla c)$, it is associated with a stochastic ItoLangevin equation

$$
\frac{d \mathbf{r}}{d t}=\chi(\rho) \nabla c+\sqrt{2 D(\rho)} \mathbf{R}(t)
$$

with

$$
\chi(\rho)=\frac{\chi g(\rho)}{\rho}, \quad D(\rho)=\frac{D}{\rho} \int_{0}^{\rho} h(x) d x,
$$

where $\mathbf{R}(t)$ is a white noise satisfying $\langle\mathbf{R}(t)\rangle=\mathbf{0}$ and $\left\langle R_{i}(t) R_{j}\left(t^{\prime}\right)\right\rangle=$ $\delta_{i j} \delta\left(t-t^{\prime}\right)$ where $i=1, \ldots, d$ label the coordinates of space. The standard Keller-Segel model is obtained when the mobility $\chi$ and the diffusion coefficient $D$ are constant. This corresponds to $h(\rho)=1$ and $g(\rho)=\rho$. In that case, the stochastic process (7) and the Fokker-Planck equation (3) are similar to the ordinary Langevin and Smoluchowski equations describing the diffusion of a system of particles in a potential $\Phi(\mathbf{r}, t)=-c(\mathbf{r}, t)$ that they 
produce themselves through Eq. (4). For example, when Eq. (4) is approximated by Eq. (6), the system becomes isomorphic to the SmoluchowskiPoisson system describing self-gravitating Brownian particles [21,22]. The steady state of the standard Keller-Segel equation is $\rho \sim e^{\frac{\chi}{D} c}$. This is similar to the Boltzmann distribution $\rho \sim e^{-\Phi / T}$ of statistical equilibrium provided that we introduce an effective temperature $T$ through the Einstein relation $T=D / \chi$. In the present study, we shall consider more general situations and allow the mobility $\chi(\rho)$ and the diffusion coefficient $D(\rho)$ to depend on the local concentration of particles $\rho(\mathbf{r}, t)$. This is an heuristic approach to take into account microscopic constraints that affect the dynamics of the particles at small scales and lead to non-Boltzmannian distributions at equilibrium. Indeed, it is not surprising that the mobility or the diffusive properties of a particle depend on its environement. For example, in a dense medium its motion can be hampered by the presence of the other particles so that its mobility is reduced.

\subsection{Generalized free energy and $H$-theorem}

We define the energy by

$$
E=\frac{1}{2 \lambda} \int\left[(\nabla c)^{2}+k^{2} c^{2}\right] d \mathbf{r}-\int \rho c d \mathbf{r} .
$$

For $\epsilon=0$, this expression reduces to

$$
E=-\frac{1}{2} \int \rho c d \mathbf{r}
$$

On the other hand, we define the temperature by

$$
T=\frac{D}{\chi} \text {. }
$$

Therefore, the Einstein relation is preserved in the generalized thermodynamical framework. We also set $\beta=1 / T$. We introduce the generalized entropic functional

$$
S=-\int C(\rho) d \mathbf{r}
$$

where $C(\rho)$ is a convex function $\left(C^{\prime \prime} \geq 0\right)$ defined by

$$
C^{\prime \prime}(\rho)=\frac{h(\rho)}{g(\rho)} .
$$

This defines the entropy up to a term of the form $A M+B$ where $M=\int \rho d \mathbf{r}$ is the mass (which is a conserved quantity). We can adapt the values of the 
constants $A$ and $B$ in order to obtain convenient expressions of the entropy. Finally, we introduce the generalized free energy

$$
F=E-T S
$$

The definition of the free energy (Legendre transform) is preserved in the generalized thermodynamical framework. The free energy is the correct thermodynamical potential since the system is dissipative. Thus, it must be treated within the canonical ensemble $[5,36]$.

A straightforward calculation shows that

$$
\dot{F}=-\frac{1}{\lambda \epsilon} \int\left(-\Delta c+k^{2} c-\lambda \rho\right)^{2} d \mathbf{r}-\int \frac{1}{\chi g(\rho)}(D h(\rho) \nabla \rho-\chi g(\rho) \nabla c)^{2} d \mathbf{r} .
$$

For $\epsilon=0$, this equation reduces to

$$
\dot{F}=-\int \frac{1}{\chi g(\rho)}(D h(\rho) \nabla \rho-\chi g(\rho) \nabla c)^{2} d \mathbf{r} .
$$

Therefore, $\dot{F} \leq 0$ (in all the paper, we assume that $\epsilon, \lambda, \chi, D, g, h$ are positive quantities). This forms an $H$ theorem in the canonical ensemble $[5,36]$ for the nonlinear mean field Fokker-Planck equation (3). This also shows that the free energy $F[\rho, c]$ is the Lyapunov functional of the generalized Keller-Segel model (3)-(4). It is sometimes useful to introduce the Massieu function

$$
J=S-\beta E,
$$

which is related to the free energy by $J=-\beta F$. Clearly, we have $\dot{J} \geq 0$. We can now consider particular cases: if $D=0$ (leading to $T=0$ ), we get $F=E$ so that $\dot{E} \leq 0$. If $\chi=0$ (leading to $\beta=0$ ), we have $J=S$ so that $\dot{S} \geq 0$.

\subsection{Stationary solution}

The steady state of Eq. (3) satisfies $\dot{F}=0$. According to Eq. (15), this leads to

$$
\Delta c-k^{2} c=-\lambda \rho, \quad D h(\rho) \nabla \rho-\chi g(\rho) \nabla c=\mathbf{0} .
$$

Using Eqs. (11) and (13), the second equation can be rewritten

$$
C^{\prime \prime}(\rho) \nabla \rho-\beta \nabla c=\mathbf{0},
$$

which can be integrated into

$$
C^{\prime}(\rho)=\beta c-\alpha,
$$


where $\alpha$ is a constant of integration. Since $C$ is convex, this equation can be reversed to give

$$
\rho(\mathbf{r})=F(-\beta c(\mathbf{r})+\alpha),
$$

where $F(x)=\left(C^{\prime}\right)^{-1}(-x)$ is a monotonically decreasing function. Thus, in the steady state, the density is a monotonically increasing function $\rho=\rho(c)$ of the concentration. We have the identity

$$
\rho^{\prime}(c)=\frac{\beta}{C^{\prime \prime}(\rho)} .
$$

Substituting Eq. (21) in Eq. (5), valid for a stationary state, we obtain a mean-field equation of the form

$$
-\Delta c+k^{2} c=\lambda F(-\beta c+\alpha) .
$$

The constant of integration $\alpha$ is determined by the total mass $M$ (which is a conserved quantity). Finally, we note that the generalized entropy (12) is related to the distribution (21) by:

$$
C(\rho)=-\int^{\rho} F^{-1}(x) d x .
$$

Equation (21) determines the distribution $\rho(\mathbf{r})$ from the entropy $S$ and Eq. (24) determines the entropy from the density.

\subsection{Minimum of free energy}

The critical points of free energy at fixed mass are determined by the variational problem

$$
\delta F+T \alpha \delta M=0,
$$

where $\alpha$ is a Lagrange multiplier. We can easily establish that

$$
\begin{gathered}
\delta E=-\frac{1}{\lambda} \int\left(\Delta c-k^{2} c+\lambda \rho\right) \delta c d \mathbf{r}-\int c \delta \rho d \mathbf{r}, \\
\delta S=-\int C^{\prime}(\rho) \delta \rho d \mathbf{r} .
\end{gathered}
$$

The variational problem (25) then leads to

$$
\Delta c-k^{2} c=-\lambda \rho, \quad C^{\prime}(\rho)=\beta c-\alpha .
$$

Comparing with Eq. (20), we find that a stationary solution of Eq. (3) is a critical point of $F$ at fixed mass. On the other hand, we have established that

$$
\dot{F} \leq 0, \quad \dot{F}=0 \Leftrightarrow \partial_{t} \rho=0 .
$$


According to Lyapunov's direct method [4], this implies that $\rho(\mathbf{r})$ is linearly dynamically stable with respect to the NFP equation (3)-(4) iff it is a (local) minimum of $F$ at fixed mass. Maxima or saddle points of $F$ are dynamically unstable. In conclusion, a steady solution of the GKS model/NFP equation (3)-(4) is linearly dynamically stable iff it satisfies (at least locally) the minimization problem:

$$
\min _{\rho, c}\{F[\rho, c] \quad \mid \quad M[\rho]=M\} .
$$

In this sense, dynamical and generalized thermodynamical stability in the canonical ensemble coincide. Furthermore, if $F$ is bounded from below a, we can conclude from Lyapunov's theory that the system will converge to a stable steady state of the GKS model for $t \rightarrow+\infty$.

Finally, we note that the GKS model can be written

$$
\frac{\partial \rho}{\partial t}=\nabla \cdot\left[\chi g(\rho) \nabla \frac{\delta F}{\delta \rho}\right]
$$

where $\delta / \delta \rho$ is the functional derivative. This shows that the diffusion current $\mathbf{J}=-\chi g(\rho) \nabla(\delta F / \delta \rho)$ is proportional to the gradient of a quantity $\delta F / \delta \rho$ that is uniform at equilibrium $\left((\delta F / \delta \rho)_{e q}=-T \alpha\right.$ according to Eq. (25)). This corresponds to the linear thermodynamics of Onsager. The same result can also be obtained from a generalized Maximum Free Energy Dissipation (MFED) principle which is the variational formulation of Onsager's linear thermodynamics [5].

\subsection{Particular cases}

If we take $h(\rho)=1$ and $g(\rho)=1 / C^{\prime \prime}(\rho)$, the NFP equation (3) becomes

$$
\frac{\partial \rho}{\partial t}=\nabla \cdot\left(D \nabla \rho-\frac{\chi}{C^{\prime \prime}(\rho)} \nabla c\right) .
$$

In that case, we have a constant diffusion $D(\rho)=D$ and a density dependent mobility $\chi(\rho)=\chi /\left(\rho C^{\prime \prime}(\rho)\right)$. If we take $g(\rho)=\rho$ and $h(\rho)=\rho C^{\prime \prime}(\rho)$, the NFP equation (3) becomes

$$
\frac{\partial \rho}{\partial t}=\nabla \cdot\left(D \rho C^{\prime \prime}(\rho) \nabla \rho-\chi \rho \nabla c\right) .
$$

\footnotetext{
${ }^{a}$ We note that for the standard Keller-Segel model, or for the Smoluchowski-Poisson system, the free energy is not bounded from below. In that case, the system can either relax towards a local minimum of $F$ at fixed mass (when it exists) or collapse to a Dirac peak [24], leading to a divergence of the free energy $F(t) \rightarrow-\infty$. The selection depends on a complicated basin of attraction. The same situation (basin of attraction) happens when there exists several minima of free energy at fixed mass.
} 
In that case, we have a constant mobility $\chi(\rho)=\chi$ and a density dependent diffusion $D(\rho)=D \rho[C(\rho) / \rho]^{\prime}$. Note that the condition $D(\rho) \geq 0$ requires that $[C(\rho) / \rho]^{\prime} \geq 0$. This gives a constraint on the possible forms of $C(\rho)$.

Finally, if we multiply the diffusion term and the drift term in the NFP equation (3) by the same positive function $\lambda(\mathbf{r}, t)$ (which can be for example a function of $\rho(\mathbf{r}, t)$ ), we obtain a NFP equation having the same free energy (i.e. satisfying an $H$-theorem $\dot{F} \leq 0$ ) and the same equilibrium states as the original one. Therefore, for a given entropy $C(\rho)$, we can form an infinite class of NFP equations possessing the same general properties [5].

\subsection{Generalized Smoluchowski equation}

The NFP equation (33) can be written in the form of a generalized Smoluchowski (GS) equation

$$
\frac{\partial \rho}{\partial t}=\nabla \cdot[\chi(\nabla p-\rho \nabla c)],
$$

with a barotropic equation of state $p(\rho)$ given by

$$
p^{\prime}(\rho)=T \rho C^{\prime \prime}(\rho) \text {. }
$$

Since $C$ is convex, we have $p^{\prime}(\rho) \geq 0$. Integrating Eq. (35) twice, we get

$$
T C(\rho)=\rho \int^{\rho} \frac{p\left(\rho^{\prime}\right)}{\rho^{\prime 2}} d \rho^{\prime} .
$$

Therefore, the free energy (14) can be rewritten

$$
F=\frac{1}{2 \lambda} \int\left[(\nabla c)^{2}+k^{2} c^{2}\right] d \mathbf{r}-\int \rho c d \mathbf{r}+\int \rho \int^{\rho} \frac{p\left(\rho^{\prime}\right)}{\rho^{\prime 2}} d \rho^{\prime} d \mathbf{r} .
$$

With these notations, the $H$-theorem becomes

$$
\dot{F}=-\frac{1}{\lambda \epsilon} \int\left(\Delta c-k^{2} c+\lambda \rho\right)^{2} d \mathbf{r}-\int \frac{1}{\chi \rho}(\nabla p-\rho \nabla c)^{2} d \mathbf{r} \leq 0 .
$$

The stationary solutions of the GS equation (34) satisfy the relation

$$
\nabla p-\rho \nabla c=\mathbf{0},
$$

which is similar to a condition of hydrostatic equilibrium. Since $p=p(\rho)$, this relation can be integrated to give $\rho=\rho(c)$ through

$$
\int^{\rho} \frac{p^{\prime}\left(\rho^{\prime}\right)}{\rho^{\prime}} d \rho^{\prime}=c .
$$

This is equivalent to

$$
\frac{p^{\prime}(\rho)}{\rho}=\frac{1}{\rho^{\prime}(c)}
$$


This relation can also be obtained from Eqs. (35) and (22). Therefore, we recover the fact that, in the steady state, $\rho=\rho(c)$ is a monotonically increasing function of $c$. We also note the identity

$$
p(\rho)=\frac{1}{\chi} D(\rho) \rho=T \rho^{2}\left[\frac{C(\rho)}{\rho}\right]^{\prime}=T\left[C^{\prime}(\rho) \rho-C(\rho)\right] .
$$

Finally, we note that the relation (40) can also be obtained by extremizing the free energy (37) at fixed mass writing $\delta F-\alpha \delta M=0$. More precisely, we have the important result: a steady solution of the generalized Smoluchowski equation (34)-(4) is linearly dynamically stable iff it is a (local) minimum of the free energy $F[\rho, c]$ at fixed mass $M[\rho]=M$. This corresponds to the minimization problem (30).

The generalized Smoluchowski equation (34) can also be obtained formally from the damped Euler equations [5]:

$$
\begin{gathered}
\frac{\partial \rho}{\partial t}+\nabla \cdot(\rho \mathbf{u})=0 \\
\frac{\partial \mathbf{u}}{\partial t}+(\mathbf{u} \cdot \nabla) \mathbf{u}=-\frac{1}{\rho} \nabla p+\nabla c-\xi \mathbf{u} .
\end{gathered}
$$

For $\xi=0$, we recover the usual barotropic Euler equations of hydrodynamics. Alternatively, if we consider the strong friction limit $\xi \rightarrow+\infty$, we can formally neglect the inertial term in Eq. (44) and we get $\xi \mathbf{u}=$ $-\frac{1}{\rho} \nabla p+\nabla c+O\left(\xi^{-1}\right)$. Substituting this relation in the continuity equation (43), we obtain the generalized Smoluchowski equation (34) with $\chi=1 / \xi$. These hydrodynamic equations (hyperbolic model) have been proposed in the context of chemotaxis to describe the organization of endothelial cells [6,37-39]. This inertial model takes into account the fact that the cells do not respond immediately to the chemotactic drift but that they have the tendency to continue in a given direction on their own. Therefore, the inertial term models cells directional persistence while the general density dependent pressure term $-\nabla p(\rho)$ takes into account anomalous diffusion or the fact that the cells do not interpenetrate. Finally, the friction force $-\xi \mathbf{u}$ measures the response of the system to the chemotactic "force" $\nabla c$. Indeed, after a relaxation time of the order $\xi^{-1}$ their velocity will be aligned with the chemotactic gradient. For $\xi=0$, Eqs. (43)-(44) lead to the formation of filaments that are interpreted as the beginning of a vasculature [6,37-39]. These filaments, or networks patterns, are not obtained in the Keller-Segel model (parabolic model), corresponding to $\xi \rightarrow+\infty$, which leads to pointwise blow up or round aggregates [7,23]. Note finally, that the GS equation 
(34) can be derived rigorously from kinetic models in a strong friction limit $\xi \rightarrow+\infty$, using a Chapman-Enskog expansion [40] or a method of moments [6].

\subsection{Kinetic derivation of the generalized Keller-Segel model}

As discussed previously, the generalized Keller-Segel model (3) can be viewed as a nonlinear Fokker-Planck equation where the diffusion coefficient and the mobility explicitly depend on the local concentration of particles. Such generalized Fokker-Planck equations can be derived from a kinetic theory, starting from the master equation, and assuming that the probabilities of transition explicitly depend on the occupation number (concentration) of the initial and arrival states. Below, we briefly summarize and adapt to the present situation the approach developed by Kaniadakis [41] in a more general context.

We introduce a stochastic dynamics by defining the probability of transition of a particle from position $\mathbf{r}$ to position $\mathbf{r}^{\prime}$. Following Kaniadakis [41], we assume the following form

$$
\pi\left(\mathbf{r} \rightarrow \mathbf{r}^{\prime}\right)=w\left(\mathbf{r}, \mathbf{r}-\mathbf{r}^{\prime}\right) a[\rho(\mathbf{r}, t)] b\left[\rho\left(\mathbf{r}^{\prime}, t\right)\right] .
$$

Usual stochastic processes correspond to $a(\rho)=\rho$ and $b(\rho)=1$ : the probability of transition is proportional to the density of the initial state and independent on the density of the final state. They lead to the ordinary Fokker-Planck equation (64) as will be shown below. Here, we assume a more general dependence on the occupancy in the initial and arrival states. This can account for microscopic constraints like close-packing effects that can inhibitate the transition. Quite generally, the evolution of the density satisfies the master equation

$$
\frac{\partial \rho}{\partial t}=\int\left[\pi\left(\mathbf{r}^{\prime} \rightarrow \mathbf{r}\right)-\pi\left(\mathbf{r} \rightarrow \mathbf{r}^{\prime}\right)\right] d \mathbf{r}^{\prime} .
$$

Assuming that the evolution is sufficiently slow, and local, such that the dynamics only permits values of $\mathbf{r}^{\prime}$ close to $\mathbf{r}$, one can develop the term in brackets in Eq. (46) in powers of $\mathbf{r}-\mathbf{r}^{\prime}$. Proceeding along the lines of [41], we obtain a Fokker-Planck-like equation

$$
\frac{\partial \rho}{\partial t}=\frac{\partial}{\partial x_{i}}\left[\left(\zeta_{i}+\frac{\partial \zeta_{i j}}{\partial x_{j}}\right) \gamma(\rho)+\gamma(\rho) \frac{\partial \ln \kappa(\rho)}{\partial \rho} \zeta_{i j} \frac{\partial \rho}{\partial x_{j}}\right],
$$

with

$$
\gamma(\rho)=a(\rho) b(\rho), \quad \kappa(\rho)=\frac{a(\rho)}{b(\rho)},
$$


and

$$
\begin{aligned}
\zeta_{i}(\mathbf{r}) & =-\int y_{i} w(\mathbf{r}, \mathbf{y}) d \mathbf{y}, \\
\zeta_{i j}(\mathbf{r}) & =\frac{1}{2} \int y_{i} y_{j} w(\mathbf{r}, \mathbf{y}) d \mathbf{y} .
\end{aligned}
$$

The moments $\zeta_{i}$ and $\zeta_{i j}$ are fixed by the Langevin equation

$$
\frac{d \mathbf{r}}{d t}=\chi \nabla c+\sqrt{2 D} \mathbf{R}(t) .
$$

Assuming isotropy $\zeta_{i}=J_{i}, \zeta_{i j}=D \delta_{i j}$, the kinetic equation (47) becomes

$$
\frac{\partial \rho}{\partial t}=\nabla \cdot\left[(\mathbf{J}+\nabla D) \gamma(\rho)-\gamma(\rho) \frac{\partial \ln \kappa(\rho)}{\partial \rho} D \nabla c\right] .
$$

Now, according to the Langevin equation (51), $D$ is independent on $\mathbf{r}$ and $\mathbf{J}=-\chi \nabla c$. Thus, we get

$$
\frac{\partial \rho}{\partial t}=\nabla \cdot\left[D \gamma(\rho) \frac{\partial \ln \kappa(\rho)}{\partial \rho} \nabla \rho-\chi \gamma(\rho) \nabla c\right] .
$$

If we define

$$
h(\rho)=\gamma(\rho) \frac{\partial \ln \kappa(\rho)}{\partial \rho}, \quad g(\rho)=\gamma(\rho),
$$

the foregoing equation can be rewritten

$$
\frac{\partial \rho}{\partial t}=\nabla \cdot(D h(\rho) \nabla \rho-\chi g(\rho) \nabla c),
$$

and it coincides with the GKS model (3). We note that

$$
\ln \kappa(\rho)=C^{\prime}(\rho) .
$$

We also have the relations

$$
\begin{gathered}
a(\rho)=\sqrt{\gamma(\rho) \kappa(\rho)}=\sqrt{g(\rho)} e^{C^{\prime}(\rho) / 2}, \\
b(\rho)=\sqrt{\frac{\gamma(\rho)}{\kappa(\rho)}}=\sqrt{g(\rho)} e^{-C^{\prime}(\rho) / 2} .
\end{gathered}
$$

Inversely,

$$
\begin{gathered}
g(\rho)=a(\rho) b(\rho), \quad C^{\prime}(\rho)=\ln \left[\frac{a(\rho)}{b(\rho)}\right], \\
h(\rho)=b(\rho) a^{\prime}(\rho)-a(\rho) b^{\prime}(\rho) .
\end{gathered}
$$


It seems natural to assume that the transition probability is proportional to the density of the initial state so that $a(\rho)=\rho$. In that case, we obtain an equation of the form

$$
\frac{\partial \rho}{\partial t}=\nabla \cdot\left(D\left[b(\rho)-\rho b^{\prime}(\rho)\right] \nabla \rho-\chi \rho b(\rho) \nabla c\right) .
$$

Note that the coefficients of diffusion and mobility are not independent since they are both expressed in terms of $b(\rho)$. Choosing $b(\rho)=1$, i.e. a probability of transition which does not depend on the population of the arrival state, leads to the standard Fokker-Planck equation, or standard Keller-Segel model (64). If, now, we assume that the transition probability is blocked (inhibited) if the concentration of the arrival state is equal to an upper bound $\sigma_{0}$, then it seems natural to take $b(\rho)=1-\rho / \sigma_{0}$. In that case, we obtain

$$
\frac{\partial \rho}{\partial t}=\nabla \cdot\left(D \nabla \rho-\chi \rho\left(1-\rho / \sigma_{0}\right) \nabla c\right),
$$

which will be considered in Sec. 3.5. Inversely, we can wonder what the general form of the mobility will be if we assume a normal diffusion $h(\rho)=1$. This leads to $b(\rho)-\rho b^{\prime}(\rho)=1$ which is integrated in $b(\rho)=1+K \rho$ where $K$ is a constant. Interestingly, we find that this condition selects the class of fermions $(K=-1)$ and bosons $(K=+1)$ and intermediate statistics (arbitrary $K$ ). The corresponding NFP equation is

$$
\frac{\partial \rho}{\partial t}=\nabla \cdot(D \nabla \rho-\chi \rho(1+K \rho) \nabla c) .
$$

\section{Examples of generalized Keller-Segel models}

In this section, we consider generalized Keller-Segel models of chemotaxis and show their relation with a formalism of generalized thermodynamics.

\subsection{The standard Keller-Segel model: Boltzmann entropy}

If we take $h(\rho)=1$ and $g(\rho)=\rho$, we get the standard Keller-Segel model

$$
\frac{\partial \rho}{\partial t}=\nabla \cdot(D \nabla \rho-\chi \rho \nabla c) .
$$

It corresponds to an ordinary diffusion $D(\rho)=D$ and a constant mobility $\chi(\rho)=\chi$. The associated stochastic process is the ordinary Langevin equation

$$
\frac{d \mathbf{r}}{d t}=\chi \nabla c+\sqrt{2 D} \mathbf{R}(t)
$$


The entropy is the Boltzmann entropy

$$
S=-\int \rho \ln \rho d \mathbf{r},
$$

and the stationary solution of Eq. (64) is the Boltzmann distribution

$$
\rho=e^{\beta c-\alpha-1} \text {. }
$$

The standard Keller-Segel model is isomorphic to the Smoluchowski equation with an isothermal equation of state

$$
p(\rho)=\rho T \text {. }
$$

\subsection{Generalized Keller-Segel model with power law diffusion: Tsallis entropy}

If we take $h(\rho)=q \rho^{q-1}$ and $g(\rho)=\rho$, we obtain the GKS model

$$
\frac{\partial \rho}{\partial t}=\nabla \cdot\left(D \nabla \rho^{q}-\chi \rho \nabla c\right) .
$$

It corresponds to a power law diffusion $D(\rho)=D \rho^{q-1}$ and a constant mobility $\chi(\rho)=\chi$. The associated stochastic process is

$$
\frac{d \mathbf{r}}{d t}=\chi \nabla c+\sqrt{2 D} \rho^{\frac{q-1}{2}} \mathbf{R}(t) .
$$

This model can take into account effects of non-ergodicity and nonextensivity. It leads to a situation of anomalous diffusion related to the Tsallis statistics [33]. For $q=1$, we recover the standard Keller-Segel model with a constant diffusion coefficient, corresponding to a pure random walk (Brownian model). In that case, the sizes of the random kicks are uniform and do not depend on where the particle happens to be. For $q \neq 1$, the size of the random kicks changes, depending on the distribution of the particles around the "test" particle. A particle which is in a region that is highly populated [large $\rho(\mathbf{r}, t)$ ] will tend to have larger kicks if $q>1$ and smaller kicks if $q<1$. Since the microscopics depends on the actual density in phase space, this creates a bias in the ergodic behavior of the system. Then, the dynamics has a fractal or multi-fractal phase space structure. The generalized entropy associated to Eq. (69) is the Tsallis entropy

$$
S=-\frac{1}{q-1} \int\left(\rho^{q}-\rho\right) d \mathbf{r},
$$

and the stationary solution is the Tsallis distribution

$$
\rho=\left(\frac{1}{q}\right)^{\frac{1}{q-1}}[1-(q-1)(-\beta c+\alpha)]_{+}^{1 /(q-1)} .
$$


The generalized Keller-Segel model (69) is isomorphic to the generalized Smoluchowski equation (34) with an equation of state

$$
p(\rho)=T \rho^{q} .
$$

This is similar to a polytropic gas with an equation of state $p=K \rho^{\gamma}$ (with $\gamma=1+1 / n$ ) where $K=T$ plays the role of a polytropic temperature and $q=\gamma$ is the polytropic index. For $q=1$, we recover the standard KellerSegel model (64). For $q=2$, we have some simplifications. In that case, the GKS model (69) becomes

$$
\frac{\partial \rho}{\partial t}=\nabla \cdot\left(D \nabla \rho^{2}-\chi \rho \nabla c\right) .
$$

The entropy is the quadratic functional

$$
S=-\int \rho^{2} d \mathbf{r},
$$

and the stationary solution is

$$
\rho=-\frac{1}{2}(-\beta c+\alpha)
$$

corresponding to a linear relation between the density and the concentration. In that case, the differential equation (23) determining the steady state reduces to the Helmholtz equation. Finally, the pressure is

$$
p(\rho)=T \rho^{2} .
$$

In the context of generalized thermodynamics, the NFP equation (69) was introduced by Plastino \& Plastino [42] and the generalized stochastic process (70) was introduced by Borland [43]. When the NFP equation (69) is coupled to the Poisson equation (6), we obtain the polytropic Smoluchowski Poisson system describing self-gravitating Langevin particles. When the NFP equation (69) is coupled to the field Eq. (4), we obtain a generalized Keller-Segel model of chemotaxis taking into account anomalous diffusion. These models have been introduced and studied by Chavanis \& Sire [6,32] For the particular index $n_{3}=d /(d-2)$ or $q_{4 / 3}=\gamma_{4 / 3}=2(d-1) / d$, the GKS model presents a critical dynamics [34].

\subsection{Generalized Keller-Segel model with logarithmic diffusion: logotropes}

If we take $h(\rho)=1 / \rho$ and $g(\rho)=\rho$, we obtain a GKS model with a logarithmic diffusion

$$
\frac{\partial \rho}{\partial t}=\nabla \cdot(D \nabla \ln \rho-\chi \rho \nabla c) .
$$


The generalized entropy associated to Eq. (78) is the log-entropy

$$
S=\int \ln \rho d \mathbf{r}
$$

and the stationary solution is

$$
\rho=\frac{1}{\alpha-\beta c} .
$$

The pressure law is

$$
p(\rho)=T \ln \rho .
$$

This is similar to a logotropic equation of state [44]. This is also connected to a polytropic equation of state (or Tsallis distribution) with $\gamma=q=0$. Indeed, the logotropic model (78) can be deduced from Eq. (69) by writing $D \nabla \rho^{q}=D q \rho^{q-1} \nabla \rho$, taking $q=0$ and re-defining $D q \rightarrow D$. When the NFP equation (78) is coupled to the Poisson equation (6), we obtain the logotropic Smoluchowski-Poisson system. When the NFP equation (78) is coupled to the field Eq. (4), we obtain a generalized Keller-Segel model of chemotaxis. These models have been introduced and studied by Chavanis $\&$ Sire [35].

\subsection{Generalized Keller-Segel models with power law diffusion and power law drift: Tsallis entropy}

We introduce here an extension of the GKS model (69). If we take $h(\rho)=$ $q \rho^{q+\mu-1}$ and $g(\rho)=\rho^{\mu+1}$, we obtain

$$
\frac{\partial \rho}{\partial t}=\nabla \cdot\left(D q \rho^{q+\mu-1} \nabla \rho-\chi \rho^{\mu+1} \nabla c\right) .
$$

This corresponds to a power law diffusion $D(\rho)=\frac{D q}{q+\mu} \rho^{q+\mu-1}$ and a power law mobility $\chi(\rho)=\chi \rho^{\mu}$. The associated stochastic process is

$$
\frac{d \mathbf{r}}{d t}=\chi \rho^{\mu} \nabla c+\sqrt{\frac{2 D q}{q+\mu}} \rho^{\frac{q+\mu-1}{2}} \mathbf{R}(t) .
$$

Since $\rho^{\mu}$ can be put in factor of the diffusion current in Eq. (82), this model has the same equilibrium states (72) and the same entropy (71) as Eq. (69).

For $\mu=0$, we recover Eq. (69) with a constant mobility and a power law diffusion. For $(\mu, q)=(0,0)$, we recover the logotropic Smoluchowski equation (78) provided that we make the transformation $D q \rightarrow D$. For $\mu=1-q$, we have a normal diffusion and a power law mobility

$$
\frac{\partial \rho}{\partial t}=\nabla \cdot\left(D q \nabla \rho-\chi \rho^{2-q} \nabla c\right) .
$$


For $q=2$, we get

$$
\frac{\partial \rho}{\partial t}=\nabla \cdot(2 D \nabla \rho-\chi \nabla c),
$$

which has the same entropy and the same equilibrium states as Eq. (74). Finally, for $q=0$ (making the transformation $q D \rightarrow D$ ), we obtain

$$
\frac{\partial \rho}{\partial t}=\nabla \cdot\left(D \nabla \rho-\chi \rho^{2} \nabla c\right),
$$

which has the same entropy and the same equilibrium states as Eq. (78). When the NFP equation (82) is coupled to the field equation (4), we obtain a generalized Keller-Segel model of chemotaxis taking into account anomalous diffusion and anomalous mobility.

\subsection{Generalized Keller-Segel models with a filling factor: Fermi-Dirac entropy}

If we take $h(\rho)=1$ and $g(\rho)=\rho\left(1-\rho / \sigma_{0}\right)$, we obtain a GKS model of the form

$$
\frac{\partial \rho}{\partial t}=\nabla \cdot\left(D \nabla \rho-\chi \rho\left(1-\rho / \sigma_{0}\right) \nabla c\right) .
$$

This corresponds to a normal diffusion $D(\rho)=D$ and a mobility $\chi(\rho)=$ $\chi\left(1-\rho / \sigma_{0}\right)$ vanishing linearly when the density reaches the maximum value $\rho_{\max }=\sigma_{0}$. The associated stochastic process is

$$
\frac{d \mathbf{r}}{d t}=\chi\left(1-\rho / \sigma_{0}\right) \nabla c+\sqrt{2 D} \mathbf{R}(t) .
$$

The generalized entropy associated with Eq. (87) is a Fermi-Dirac-like entropy in physical space

$$
S=-\sigma_{0} \int\left\{\frac{\rho}{\sigma_{0}} \ln \frac{\rho}{\sigma_{0}}+\left(1-\frac{\rho}{\sigma_{0}}\right) \ln \left(1-\frac{\rho}{\sigma_{0}}\right)\right\} d \mathbf{r},
$$

and the stationary solution is a Fermi-Dirac-like distribution in physical space

$$
\rho=\frac{\sigma_{0}}{1+e^{-\beta c+\alpha}} .
$$

From Eq. (90), we see that, in the stationary state, $\rho<\sigma_{0}$. This bound is similar to the Pauli exclusion principle in quantum mechanics. In fact, we can show that $\rho(\mathbf{r}, t)$ remains bounded by $\sigma_{0}$ during the whole evolution. For $\sigma_{0} \rightarrow+\infty$, we recover the standard KS model (64). 
An alternative GKS model, with the same entropy and the same equilibrium states, is obtained by taking $h(\rho)=1 /\left(1-\rho / \sigma_{0}\right)$ and $g(\rho)=\rho$. This leads to

$$
\frac{\partial \rho}{\partial t}=\nabla \cdot\left(-D \sigma_{0} \nabla \ln \left(1-\rho / \sigma_{0}\right)-\chi \rho \nabla c\right) .
$$

This corresponds to a nonlinear diffusion with $D(\rho)=-\sigma_{0}(D / \rho) \ln (1-$ $\left.\rho / \sigma_{0}\right)$ and a constant mobility $\chi(\rho)=\chi$. Equation (91) can be put in the form of a generalized Smoluchowski equation (34) with a pressure law

$$
p(\rho)=-T \sigma_{0} \ln \left(1-\rho / \sigma_{0}\right) .
$$

For $\rho \ll \sigma_{0}$, we recover the "isothermal" equation of state $p=\rho T$ leading to the standard Keller-Segel model (64). However, for higher densities, the equation of state is modified and the pressure diverges when $\rho \rightarrow \sigma_{0}$. This prevents the density from exceeding the maximum value $\sigma_{0}$.

The NFP equation (87) has been introduced by Kaniadakis \& Quarati [45] to describe fermionic systems and by Robert \& Sommeria [46] in the statistical mechanics of two-dimensional turbulence (see also [47]). In the context of chemotaxis, the model (87) has been introduced by Hillen \& Painter [29] and, independently, by Chavanis [5,31]. It provides a regularization of the standard Keller-Segel model preventing overcrowding, blow-up and unphysical singularities. The filling factor $\left(1-\rho / \sigma_{0}\right)$ takes into account the fact that the particles cannot interpenetrate because of their finite size $a$. Therefore, the maximum allowable density is $\sigma_{0} \sim 1 / a^{d}$. It is achieved when all the cells are packed together. In the model (87), it is assumed that the mobility vanishes when the density reaches the close packing value ( $\left.\rho \rightarrow \sigma_{0}\right)$ while the diffusion is not affected. The alternative model (91) has been introduced by Chavanis $[5,31]$. In that case, the mobility is assumed to be constant and the regularization preventing overcrowding is taken into account in the pressure law (92). We can also multiply the diffusion term and the mobility term in the NFP equation (3) by the same positive function $\lambda(\mathbf{r}, t)$ in order to obtain a more general model with the same entropy and the same equilibrium states in which both diffusion and mobility are affected by overcrowding.

\subsection{Generalized Keller-Segel models incorporating anomalous diffusion and filling factor}

The previous models focus individually on two important effects: anomalous diffusion (see Secs. 3.2-3.4) and exclusion constraints when the density becomes too large (see Sec. 3.5). Here we introduce a mixed model which 
combines these two effects in a single equation. If we take $h(\rho)=q \rho^{q+\mu-1}$ and $g(\rho)=\rho^{\mu+1}\left(1-\rho / \sigma_{0}\right)$, we obtain

$$
\frac{\partial \rho}{\partial t}=\nabla \cdot\left(D q \rho^{q+\mu-1} \nabla \rho-\chi \rho^{\mu+1}\left(1-\rho / \sigma_{0}\right) \nabla c\right) .
$$

This corresponds to a power law diffusion such that $D(\rho)=$ $[D q /(q+\mu)] \rho^{q+\mu-1}$ and a mobility $\chi(\rho)=\chi \rho^{\mu}\left(1-\rho / \sigma_{0}\right)$. The associated stochastic process is

$$
\frac{d \mathbf{r}}{d t}=\chi \rho^{\mu}\left(1-\rho / \sigma_{0}\right) \nabla c+\sqrt{\frac{2 D q}{q+\mu}} \rho^{\frac{q+\mu-1}{2}} \mathbf{R}(t) .
$$

The generalized entropy corresponding to Eq. (93) is obtained by integrating twice the relation

$$
C^{\prime \prime}(\rho)=\frac{q \rho^{q-2}}{1-\rho / \sigma_{0}}
$$

A first integration gives

$$
C^{\prime}(\rho)=q \sigma_{0}^{q-1} \Phi_{q-2}\left(\frac{\rho}{\sigma_{0}}\right),
$$

where

$$
\Phi_{m}(t)=\int_{0}^{t} \frac{x^{m}}{1-x} d x .
$$

Therefore, the generalized entropy can be expressed as

$$
C(\rho)=q \sigma_{0}^{q} \int_{0}^{\rho / \sigma_{0}} \Phi_{q-2}(t) d t .
$$

On the other hand, the equilibrium density is given by $\rho=\sigma_{0} \Phi_{q-2}^{-1}[(\beta c-$ $\left.\alpha) / q \sigma_{0}^{q-1}\right]$. Note that these results not depend on $\mu$ since the term $\rho^{\mu}$ can be put in factor of the diffusion current in Eq. (93).

Let us consider some particular cases. (i) For $q=1$, Eq. (93) has the same entropy and the same equilibrium states as Eq. (87). (ii) For $\sigma_{0} \rightarrow$ $+\infty$, we recover Eq. (82). (iii) For $\mu=0$ and $q=2$, we have

$$
\frac{\partial \rho}{\partial t}=\nabla \cdot\left(D \nabla \rho^{2}-\chi \rho\left(1-\rho / \sigma_{0}\right) \nabla c\right) .
$$

The generalized entropy is

$$
S=-2 \sigma_{0}^{2} \int\left(1-\frac{\rho}{\sigma_{0}}\right) \ln \left(1-\frac{\rho}{\sigma_{0}}\right) d \mathbf{r},
$$


and the stationary solution is

$$
\rho=\sigma_{0}\left[1-e^{(-\beta c+\alpha) / 2 \sigma_{0}}\right]_{+} .
$$

For $\sigma_{0} \rightarrow+\infty$, we recover Eq. (76). Dividing the diffusion and the drift term by $1-\rho / \sigma_{0}$, we can also consider the alternative model

$$
\frac{\partial \rho}{\partial t}=\nabla \cdot\left(\frac{2 \rho D}{1-\rho / \sigma_{0}} \nabla \rho-\chi \rho \nabla c\right),
$$

which has the same entropy and the same equilibrium states as Eq. (99). The pressure law is

$$
p(\rho)=-2 T \sigma_{0}^{2}\left[\ln \left(1-\rho / \sigma_{0}\right)-\rho / \sigma_{0}\right] .
$$

(iv) For $(\mu, q)=(0,0)$ and performing the transformation $q D \rightarrow D$, or directly taking $h(\rho)=1 / \rho$ and $g(\rho)=\rho\left(1-\rho / \sigma_{0}\right)$, we obtain

$$
\frac{\partial \rho}{\partial t}=\nabla \cdot\left(D \nabla \ln \rho-\chi \rho\left(1-\rho / \sigma_{0}\right) \nabla c\right) .
$$

This corresponds to a logarithmic diffusion and a modified mobility taking into account an exclusion principle through the filling factor. The generalized entropy is obtained from the relation

$$
C^{\prime \prime}(\rho)=\frac{1}{\rho^{2}\left(1-\rho / \sigma_{0}\right)},
$$

leading to

$$
C^{\prime}(\rho)=-\frac{1}{\sigma_{0}}\left\{\ln \left(\frac{\sigma_{0}}{\rho}-1\right)+\frac{\sigma_{0}}{\rho}\right\},
$$

and finally to the explicit expression

$$
S=-\int\left(1-\frac{\rho}{\sigma_{0}}\right) \ln \left(\frac{\sigma_{0}}{\rho}-1\right) d \mathbf{r} .
$$

We can consider the alternative model

$$
\frac{\partial \rho}{\partial t}=\nabla \cdot\left[\frac{D}{\rho\left(1-\rho / \sigma_{0}\right)} \nabla \rho-\chi \rho \nabla c\right],
$$

with the same entropy and the same equilibrium states. The associated pressure law is

$$
p(\rho)=-T \ln \left(\frac{\sigma_{0}}{\rho}-1\right) .
$$




\section{Conclusion}

In this paper, we have discussed a generalized class of Keller-Segel models describing the chemotaxis of biological populations. We have shown their analogy with nonlinear mean field Fokker-Planck equations and generalized thermodynamics. We have given explicit examples corresponding to different entropy functionals. In particular, we have considered the case where the particles (cells) experience anomalous diffusion and the case where they experience an exclusion constraint (volume filling). We have introduced a mixed model taking into account these two effects in a single equation (93). Of course, we can construct other types of Keller-Segel models which may also be of interest. The general study of these models, which combine both long-range interactions and generalized thermodynamics, is very rich and can lead to a wide diversity of phase transitions and blow up phenomena. These nonlinear meanfield Fokker-Planck equations are therefore of considerable theoretical interest [5].

\section{References}

1. J.D. Murray, Mathematical Biology (Springer, Berlin, 1991).

2. C.S. Patlak, Bull. of Math. Biophys. 15, 311 (1953).

3. E.F. Keller and L.A. Segel, J. Theor. Biol. 30, 225 (1971).

4. T.D. Frank, Nonlinear Fokker-Planck Equations: Fundamentals and Applications, (Springer-Verlag, 2005).

5. P.H. Chavanis, Phys. Rev. E 68, 036108 (2003).

6. P.H. Chavanis, C. Sire, Physica A 384, 199 (2007).

7. D. Horstmann, Jahresberichte der DMV 106, 51 (2004).

8. V. Nanjundiah, J. Theoret. Biol. 42, 63 (1973).

9. S. Childress and J.K. Percus, Math. Biosci. 56, 217 (1981).

10. W. Jäger, S. Luckhaus, Trans. Amer. Math. Soc. 329, 819 (1992).

11. T. Nagai, Adv. Math. Sci. Appl. 5, 581 (1995).

12. H. G. Othmer and A. Stevens, SIAM J. Appl. Math. 57, 1044 (1997).

13. M.A. Herrero and J.L. Velazquez, Math. Ann. 306, 583 (1996).

14. M.A. Herrero, E. Medina and J.L. Velazquez, Nonlinearity 10, 1739 (1997).

15. M.A. Herrero, E. Medina, and J.L. Velazquez, J. Comput. Appl. Math. 97, 99 (1998).

16. P. Biler, Adv. Math. Sci. Appl. 8, 715 (1998).

17. M.P. Brenner, P. Constantin, L.P. Kadanoff, A. Schenkel and S.C. Venkataramani, Nonlinearity 12, 1071 (1999).

18. J. Dolbeault, B. Perthame, C. R. Acad. Sci. Paris, Ser. I 339, 611 (2004).

19. P. Biler, G. Karch, P. Laurençot, T. Nadzieja, Topol. Methods Nonlinear Anal. 27, 133 (2006).

20. P. Biler, G. Karch, P. Laurençot, T. Nadzieja, Math. Methods Appl. Sci. 29, 1563 (2006). 
21. P.H. Chavanis, C. Rosier and C. Sire, Phys. Rev. E 66, 036105 (2002).

22. P.H. Chavanis, Physica A 384, 392 (2007).

23. C. Sire and P.H. Chavanis, Phys. Rev. E 66, 046133 (2002).

24. C. Sire and P.H. Chavanis, Phys. Rev. E 69, 066109 (2004).

25. P.H. Chavanis and C. Sire, Phys. Rev. E 70, 026115 (2004).

26. J. Sopik, C. Sire and P.H. Chavanis, Phys. Rev. E 72, 026105 (2005).

27. P.H. Chavanis and C. Sire, Phys. Rev. E 73, 066103 (2006).

28. P.H. Chavanis and C. Sire, Phys. Rev. E 73, 066104 (2006).

29. T. Hillen and K. Painter, Adv. Appl. Math. 26, 280 (2001).

30. K. Painter and T. Hillen, Can. Appl. Math. Q. 10, 501 (2002).

31. P.H. Chavanis, Eur. Phys. J. B 54, 525 (2006).

32. P.H. Chavanis and C. Sire, Phys. Rev. E 69, 016116 (2004).

33. C. Tsallis, J. Stat. Phys. 52, 479 (1988).

34. P.H. Chavanis, C. Sire, [arXiv:0705.4366]

35. P.H. Chavanis, C. Sire, Physica A 375, 140 (2007).

36. P.H. Chavanis, Physica A 361, 55 (2006).

37. A. Gamba, D. Ambrosi, A. Coniglio, A. de Candia, S. di Talia, E. Giraudo, G. Serini, L. Preziosi, F. Bussolino, Phys. Rev. Lett. 90, 118101 (2003).

38. F. Filbet, P. Laurençot, B. Perthame, J. Math. Biol. 50, 189 (2005).

39. P.H. Chavanis, Eur. Phys. J. B 52, 433 (2006); P.H. Chavanis, C. Sire [arXiv:0708.3163]

40. P.H. Chavanis, P. Laurençot, M. Lemou, Physica A 341, 145 (2004).

41. G. Kaniadakis, Physica A 296, 405 (2001).

42. A.R. Plastino, A. Plastino, Physica A 222, 347 (1995).

43. L. Borland, Phys. Rev. E 57, 6634 (1998).

44. D.E. McLaughlin, R.E. Pudritz, Astrophys. J. 476, 750 (1997).

45. G. Kaniadakis, P. Quarati Phys. Rev. E 49, 5103 (1994).

46. R. Robert, J. Sommeria, Phys. Rev. Lett. 69, 2776 (1992).

47. P.H. Chavanis, J. Sommeria, R. Robert, Astrophys. J. 471, 385 (1996). 